\title{
THz time-domain spectroscopy uncertainties
}

\author{
Hungyen Lin, Withawat Withayachumnankul, Bernd M. Fischer, Samuel P. Mickan, and Derek Abbott \\ School of Electrical \& Electronic Engineering, The University of Adelaide, SA 5005, Australia \\ Email: hlin@eleceng.adelaide.edu.au
}

\begin{abstract}
THz time-domain spectroscopy (TDS) is a significant technique for material characterization as it seeks to determine the optical or dielectric constants in the T-ray regime. The precision of the constants is highly affected by the intricate THz-TDS process. Typically, a short-pulsed THz-TDS system is composed of several mechanical, optical, and electronic parts, each of which is limited in precision and therefore has an effect on the uncertainty of the achieved optical or dielectric constants. This paper analyses sources of uncertainty and models error propagation through the system.
\end{abstract}

\section{INTRODUCTION}

Terahertz time-domain spectroscopy (THz-TDS) is an important technique for material characterization. A T-ray waveform is typically transmitted through a material sample with its amplitude and phase linearly altered by material's response. Sample and reference waveforms, once converted into the frequency domain, can be processed to extract the frequencydependent optical constants and quantities [1], [2]. Despite the high SNR of T-ray signals, many sources of fluctuations and variations exist. For example, ultrafast-laser instability [3], [4], water-vapor-induced fluctuations [5], reflections [6], optical and electronic noise [7], [8]. All these fluctuations affect the reliability of the extracted optical constants. In this paper, we show the propagation of sources of uncertainty in a typical THz-TDS setup (Figure 1) to the output, and model the relation between each source variance and the output variance mathematically. The uncertainties investigated herein, are only the non-reproducible fluctuations. Any fluctuation reproducible over several measurements like water-vapor-induced fluctuations and reflections is not considered here.

\section{UNCERTAINTY PROPAGATION}

Figure 2 shows the propagation of uncertainties in the THz-TDS measurement and the parameter extraction process. Optical delay line stage positioning error affects the sampling time of an optically-gated detector. Combined with the electronic and optical noise, this contributes to the sampled T-ray pulse amplitude uncertainty. The amplitude error propagates through the Fourier transform and deconvolution stages for both reference and sample signals. Additional uncertainty is picked up during phase unwrapping. The parameter extraction process that requires knowledge of sample thickness and orientation induce further uncertainty. Finally, the error in air refractive index estimation affects the overall uncertainty of the extracted optical constants. The analytical expression for each individual source of uncertainty that contributes to optical constant variance is as follows with the notations in Table I.
Amplitude uncertainty: Given the amplitude variance of the time domain of reference and sample signals, denoted by $\sigma_{E_{\text {ref }}}^{2}(k)$ and $\sigma_{E_{\text {sam }}}^{2}(k)$, respectively, the variances in the optical constants are,

$$
\begin{aligned}
\sigma_{n, E}^{2}(\omega)= & {\left[\frac{c}{\omega d}\right]^{2}\left\{\frac{A(\omega)}{\left|E_{\mathrm{sam}}(\omega)\right|^{4}}+\frac{B(\omega)}{\left|E_{\mathrm{ref}}(\omega)\right|^{4}}\right\}, } \\
\sigma_{\alpha, E}^{2}(\omega)= & \frac{4}{d^{2}}\left\{\frac{C(\omega)}{\left|E_{\mathrm{sam}}(\omega)\right|^{4}}+\frac{D(\omega)}{\left|E_{\mathrm{ref}}(\omega)\right|^{4}}\right. \\
& \left.+\left(\frac{n(\omega)-n_{0}}{n(\omega)+n_{0}}\right)^{2} \frac{\sigma_{n, E}^{2}(\omega)}{n(\omega)^{2}}\right\}
\end{aligned}
$$

where

$$
\begin{aligned}
A(\omega) & =\sum_{k=0}^{N-1} \Im^{2}\left[E_{\mathrm{sam}}(\omega) \exp (j \omega k \tau)\right] \sigma_{E_{\mathrm{sam}}}^{2}(k), \\
B(\omega) & =\sum_{k=0}^{N-1} \Im^{2}\left[E_{\mathrm{ref}}(\omega) \exp (j \omega k \tau)\right] \sigma_{E_{\mathrm{ref}}}^{2}(k), \\
C(\omega) & =\sum_{k=0}^{N-1} \Re^{2}\left[E_{\mathrm{sam}}(\omega) \exp (j \omega k \tau)\right] \sigma_{E_{\mathrm{sam}}}^{2}(k), \\
D(\omega) & =\sum_{k=0}^{N-1} \Re^{2}\left[E_{\mathrm{ref}}(\omega) \exp (j \omega k \tau)\right] \sigma_{E_{\mathrm{ref}}}^{2}(k) .
\end{aligned}
$$

Sample thickness uncertainty: Given the sample thickness variance $\sigma_{l}^{2}$, the variances of the optical constants are

$$
\begin{aligned}
\sigma_{n, l}^{2}(\omega)= & {\left[\frac{c}{\omega l^{2}} \phi_{c}(\omega) \cos \theta_{t}\right]^{2} \sigma_{l}^{2} } \\
\sigma_{\alpha, l}^{2}(\omega)= & {\left[\frac{\alpha(\omega)}{l}\right]^{2} \sigma_{l}^{2} } \\
& +\left[\frac{2}{d n(\omega)}\left(\frac{n(\omega)-n_{0}}{n(\omega)+n_{0}}\right)\right]^{2} \sigma_{n, l}^{2}(\omega) .
\end{aligned}
$$

Sample alignment uncertainty: Given the variance of the sample alignment $\sigma_{\theta_{t}}^{2}$, the variances of the optical constants are

$$
\begin{aligned}
\sigma_{n, \theta}^{2}(\omega)= & {\left[\frac{c}{\omega d} \phi_{c}(\omega) \tan \theta_{t}\right]^{2} \sigma_{\theta_{t}}^{2} } \\
\sigma_{\alpha, \theta}^{2}(\omega)= & {\left[\alpha(\omega) \tan \theta_{t}\right]^{2} \sigma_{\theta_{t}}^{2} } \\
& +\left[\frac{2}{d n(\omega)}\left(\frac{n(\omega)-n_{0}}{n(\omega)+n_{0}}\right)\right]^{2} \sigma_{n, \theta}^{2}(\omega) .
\end{aligned}
$$




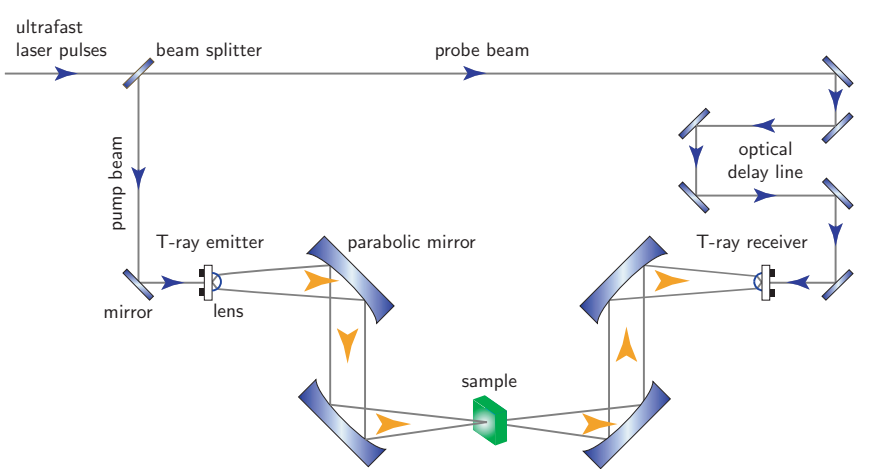

Fig. 1. THz-TDS system configured in transmission mode with PCA generation and detection. The system consists of an ultrafast optical laser, T-ray emitter/receiver, an optical delay line, a set of mirrors, and a material sample. The optical beam paths are indicated by small blue arrowheads, and the T-ray beam paths by the large orange arrowheads.

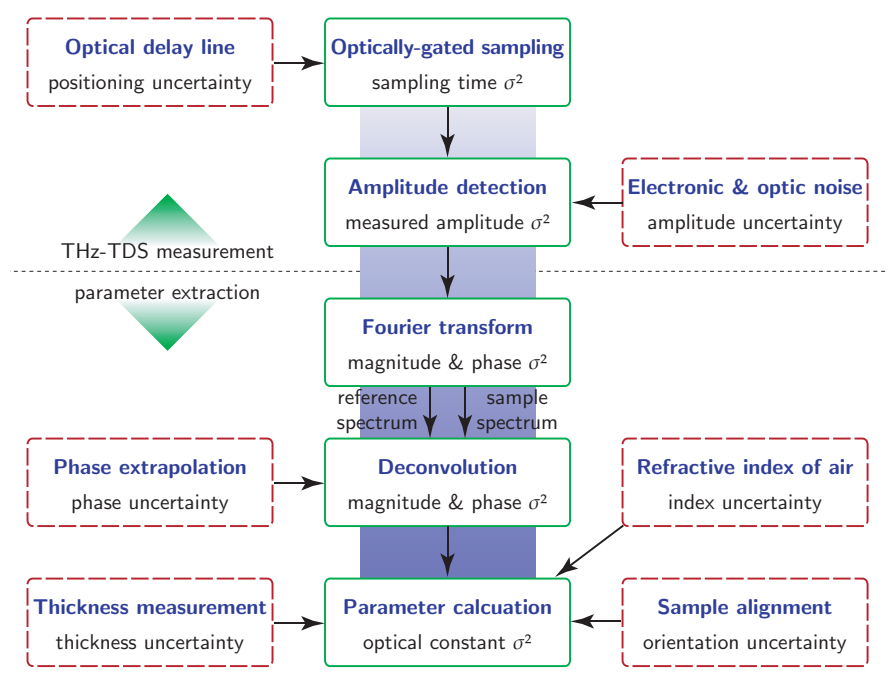

Fig. 2. Propagation of uncertainties. The uncertainty sources (red dashed lines) can take place in both the THz-TDS measurement and the parameter extraction process. They cause the variance, $\sigma^{2}$, propagating down the process, and eventually contribute to the variance in the extracted optical constants.

TABLE I

NOTATION FOR UNCERTAINTY ANALYSIS

\begin{tabular}{cl}
\hline Symbol & Description \\
\hline$c$ & speed of light in vacuum \\
$j$ & $\sqrt{-1}$ \\
$\Re^{2}$ & square of real part \\
$\Im^{2}$ & square of imaginary part \\
$\omega$ & angular frequency \\
$n_{0}$ & index of refraction of air \\
$n(\omega)$ & index of refraction of material \\
$\kappa(\omega)$ & extinction coefficient of material \\
$\alpha(\omega)$ & absorption coefficient of material, $\alpha=2 \omega \kappa / c$ \\
$l$ & thickness of material \\
$\theta_{t}$ & tilted angle of the sample \\
$d$ & path length in material, $d=l / \cos \theta_{t}$ \\
$k$ & time index, running from 0 to $N-1$ \\
$\tau$ & sampling interval \\
$E_{\text {ref }}(\omega)$ & reference signal's complex amplitude \\
$E_{\mathrm{sam}}(\omega)$ & sample signal's complex amplitude \\
$\phi_{c}(\omega)$ & $\angle E_{\text {sam }}(\omega)-\angle E_{\text {ref }}(\omega)$ \\
$\sigma_{n}^{2}(\omega)$ & variance in index of refraction \\
$\sigma_{\alpha}^{2}(\omega)$ & variance in absorption coefficient \\
\hline
\end{tabular}

Air refractive index uncertainty: Given the variance in the air refractive index $\sigma_{n_{0}}^{2}$, the variances of the optical constants are

$$
\begin{aligned}
\sigma_{n, n_{0}}^{2}(\omega) & =\sigma_{n_{0}}^{2} \\
\sigma_{\alpha, n_{0}}^{2}(\omega) & =\left[\frac{2}{d}\left(\frac{n(\omega)-n_{0}}{n(\omega)+n_{0}}\right)\right]^{2}\left[\frac{1}{n(\omega)^{2}}+\frac{1}{n_{0}^{2}}\right] \sigma_{n_{0}}^{2} .
\end{aligned}
$$

The total variance in refractive index and absorption coefficient is given by

$$
\begin{aligned}
& \sigma_{n}^{2}(\omega)=\sigma_{n, E}^{2}(\omega)+\sigma_{n, l}^{2}(\omega)+\sigma_{n, \theta}^{2}(\omega)+\sigma_{n_{0}}^{2}(\omega), \\
& \sigma_{\alpha}^{2}(\omega)=\sigma_{\alpha, E}^{2}(\omega)+\sigma_{\alpha, l}^{2}(\omega)+\sigma_{\alpha, \theta}^{2}(\omega)+\sigma_{\alpha, n_{0}}^{2}(\omega),
\end{aligned}
$$

respectively-where simple addition in quadrature is carried out as the sources of uncertainty are uncorrelated.

\section{DISCUSSION AND CONCLUSION}

This paper has presented an uncertainty analysis on THzTDS. Various sources of uncertainty in THz-TDS and the parameter extraction process are identified and modeled mathematically. In particular, the relationship between each source and the optical constants' uncertainties is considered in detail. The proposed model is applicable to either PCA or EO generation and detection systems. However, the model is firstorder approximated, which is valid for case where sources of uncertainty have small variations. Future work, will endeavor to investigate each uncertainty source in depth and provide both theoretical and practical suggestions for system optimization. A higher order analysis of uncertainty is demanded for high accuracy uncertainty estimation. Future experiments will also be carried out to substantiate the developed model.

\section{REFERENCES}

[1] L. Duvillaret, F. Garet, and J.-L. Coutaz, "A reliable method for extraction of material parameters in terahertz time-domain spectroscopy," IEEE Journal of Selected Topics in Quantum Electronics, vol. 2, no. 3, pp. 739-746, 1996.

[2] W. Withayachumnankul, B. Ferguson, T. Rainsford, S. P. Mickan, and D. Abbott, "Simple material parameter estimation via terahertz timedomain spectroscopy," IEE Electronics Letters, vol. 41, no. 14, pp. 800801, 2005.

[3] H. A. Haus and A. Mecozzi, "Noise of mode-locked lasers," IEEE Journal of Quantum Electronics, vol. 29, no. 3, pp. 983-996, 1993.

[4] A. Poppe, L. Xu, F. Krausz, and C. Spielmann, "Noise characterization of sub-10-fs Ti:sapphire oscillators," IEEE Journal of Selected Topics in Quantum Electronics, vol. 4, no. 2, pp. 179-184, 1998.

[5] M. van Exter, C. Fattinger, and D. Grischkowsky, "Terahertz time-domain spectroscopy of water vapor," Optics Letters, vol. 14, no. 20, pp. 11281130, 1989.

[6] W. Withayachumnankul, B. Ferguson, T. Rainsford, S. P. Mickan, and D. Abbott, "Direct Fabry-Pérot effect removal," Fluctuation and Noise Letters, vol. 6, no. 2, pp. L227-L239, 2006.

[7] M. van Exter and D. R. Grischkowsky, "Characterization of an optoelectronic terahertz beam system," IEEE Transactions on Microwave Theory and Techniques, vol. 38, no. 11, pp. 1684-1691, 1990.

[8] L. Duvillaret, F. Garet, and J.-L. Coutaz, "Influence of noise on the characterization of materials by terahertz time-domain spectroscopy," Journal of the Optical Society of America B: Optical Physics, vol. 17, no. 3, pp. 452-460, 2000. 